\title{
Study on Women and Literature in the Eastern Han Dynasty - A Case Study of Cai Wenji and His " Indignant Poem "
}

\author{
Hongcheng Wang \\ College of History and Culture, Northwest Normal University, China, 730070
}

Keywords: Cai Wenji, “Indignant Poem”, Poems

\begin{abstract}
This paper focuses on the poems of poems and poems written by Cai Wenji and his "poems of Indignant Poem" in the late Han Dynasty, and explores her tragic fate and literary creation to show her personality connotation and literary charm. The tragic fate of Cai Wenji reflects the tragic fate of the broad masses of society, especially women, in the warring society of the Eastern Han Dynasty, and the poems of her works have the narrative nature of self-narrative nature, which is the epitome of the whole era. "Indignant Poem poem" in the Han Dynasty and the whole history of the development of Chinese poetry among the long, have an immeasurable position, the later poetry has far-reaching impact.
\end{abstract}

\section{Introduction}

In the middle and late Eastern Han Dynasty, the rule of corruption, social contradictions intensified, the central government are all left in the hands of foreigners, eunuchs. AD 189 years, the Han Shao emperor Liu ascended the throne, how the emperor Bing Zheng, ten often chaos political affairs, the Queen Mother brother into the desire to punish eunuchs, anti-murder. Dong Zhuo by the eradication of eunuchs, to protect the emperor as an excuse to drive troops into Beijing. Immediately waste the emperor for the Hongnong Wang, Li Han Xian emperor Liu Association, change Yuan Yonghan. The world of the princes to crusade against Dong Zhuo in the name of the ghosts, the Central Plains, the battle for decades, and this will become the background and reason Cai Wenji fate.

\section{The fate of the wind swing}

Cai Wen Ji, Ming Yan, the word Zhao Ji, because the taboo Sima Zhao, and changed to Wen Ji, Chen left people. His father Cai Yong learned more proficient, proficient in calligraphy, wonderful tone law, good poetry, had served Lang Lang. She was influenced by his father, calligraphy, temperament, Ci Fu all proficient. According to Liang Liu Shao "young children pass" contains: "Yong night honeymoon, string never. Yan said: 'second string.' Yong said: 'even the ear.' There is a string asked, Yan said: The first string. 'Is not absurd. "[1]But such a peerless woman, from the birth of the unfortunate and suffering will follow.

AD 178 years ago, Cai Yong offended when the right castration, and in exile Jiangnan, Cai Wen $\mathrm{Ji}$ also followed. This is more than ten years to go. Wait until they return to the capital, the political power has been Xiliang warlords Dong Zhuo monopoly. Dong Zhuo was killed after, Cai Yong and because of "do not regret and sigh", offended Wang Yun, "that is, to pay Tingwei crime." [1] Cai Yong was regarded as Dong Zhuo party and injustice death, Cai Wenji Indignant Poem, but the fate of God did not let her tragic fate end.

Wang Yun design to kill Dong Zhuo, but unable to control the situation of the capital, so chaotic. Hu Bing took the opportunity to invade, Cai Wenji was also taken into the Southern Huns. There is only a brief record of the historical data in this event: "Xingping, the world is lost, Wen Ji for Hu riding by, not in the Southern Huns left Yin Wang." [1], "Indignant Poem" the first half of the details 
Describing the process of being captured into the Southern Huns as a result of the war, and the sufferings they suffered. She said: "Zhuozhong to the East, the golden armor of the sun." The local people are fragile, the soldiers are Hu Qiang. Hunting wild city, the cut to the cut. Cut cut no relics, corpses refused. "[1] Beginning three years (129), Dong Zhuo Department of East to exit, to resist the eighteen princes into the soldiers. At this point, Hu Bing took the opportunity to carry out the big plunder of Chen, killing open, corpses everywhere. The description of the tragic scene in the poem is the author's personal experience.

Cai Wenji captive as slaves, robbed the Southern Huns left Yin Wang Department. She said: "But the number of weeping line, the night is sad to sit. Want to die can not get, want to have no one can be" [1] This poem describes Cai Wenji was sent on the way, like pigs, sheep were driven out of the "survival Can not, beg death can not "the feelings of Indignant Poem. The so-called "cut cut no relics, corpses phase support refused", then a complete show of the Central Plains war, and Hu Binghao swept after the tragic desolate situation. "[1] captive is not considered by the people, life did not guarantee, dignity is more out of the question. She suffered a non-human experience, but had to "and then the number of weeping line, the night is sad to sit" [1]. Cai Wenji to that "side of the shortage and different, popular little reason" [1] of the Southern Huns, their very lonely lonely.

War for the people, only harm without harm. Regardless of the nature of the war, accompanied by only economic withered, the population dropped, life is only once and most valuable, and the greatest destruction of war is the lack of respect for life. Therefore, those who mind the people of the rulers, will not light war. In the war, women are not only going to be bloody and cruel as men do, but also to be looted as a booty. "Indignant Poem and indignation" for us to show a wise intellectual warfare in the eyes of the ruthless and horror, but also a new expansion of poetry and women genre.

In the context of the Wei and Jin Dynasties literary circles, many literati expressed the tragic experience of the people in the war, such as Cao Cao's "Artemisia": "Bone white in the wild, thousands of miles without chicken Ming. "[2] Wang Can poem" seven poems ":" out of nothing, the bones of the plains. Road hungry woman, holding the child to abandon the grass. Gu heard the number of sobbing, tear alone do not, 'unknown "[3] These two poems show from the different perspectives to the reader to show the scene, and the face of such a situation of Indignant Poem mood. The above two poems are typical examples of "Jian'an Style", inheriting the thought of reflecting the reality and criticizing the reality of the Han Yuefu, and the inheritance of the Han Dynasty. And Cai Wenji's "chaos from the" poem, with a witness from the female perspective, pain complained of the war that era, represents the highest point of Yuefu narrative poems.

\section{The flesh and blood of the parting}

Hu Bing laughed, Cai Wenji and her mother in the home Chen stay, and her father Cai Yong is still in the capital, so she was swept and Cai Yong injustice, the two sides did not know at first. One can imagine, a noble woman and their loved ones scattered, was taken to the incestant, unreasonable South Huns, what is the distraught. She thought innumerable death to end their own lives, but no matter what, still a trace of hope for their loved ones.

Time of the wheel in the blood and life between the rolling, is so slow and weak. In this "place more frost, Hu Fengchun Fu" from the frontier, "when the idea of parents, lamenting infinite." [1] often heard from the guests from the original to the frontier, they will not feel overjoyed, even heard that with the family and the news associated with, even if there is a relationship with Chen can, but the reality is so unreasonable.

Cai Wenji alone in the Southern Huns, the hope of a pair of parents if the count of living as a courage, her pair of love is another one. This pair of well-behaved son in her dry heart into a stream of spring, to stop her homesickness of the pain. However, she missed her homeland but want to hide something, especially in the "guest from the outside, smell the joy", [1] so forget that they have two sons. Wang Guowei in the "human words," said: "can be true scenery, true feelings, that there is a realm." [4] This sentence is used to illustrate Cai Wenji's "Indignant Poem", really appropriate. "There is a guest from the outside, smell the joy" and "encounter wish" to express her twelve years waiting for their pleasure, but also must make a difficult choice. When she was very cruel to decide 
"have been self-solution free, when the abandoned son", "children before my neck, asked the mother to what." When the words to go, how complex? Side of the present, what is more disrespectful? I have not yet adult, but why do not think? "'[1] as the mother of Cai Wenji, son of words words stung her heart, make her distraught.

Her son heard that he will return to the Central Plains, homes, The child's words enough to make a mother "see this collapse of five, trance mad mad. No. Hushao touch, when the change back to doubt." [1] The passion for the love of the two sons and the hometown of love is the source of the real pain of the poet. Cai Wenji finally reluctantly cut off the flesh and blood contact emotional ties, alone on the return journey. Every step forward, the great maternal love of the wound, will be blood dripping, life and death of mother and child love the great pain and how to heal. "The Book of Songs - Kay wind": "Kay wind from the south, blowing his spirits heart. Heartbreak, mothers and young labor. Kay wind from the south, blowing his spine wages. Mother's holy good, I have no people." [5] This is the infinite praise of the mother from the child's point of view. And Cai Wenji's "Indignant Poem and indignation" in the mother's role is her own, fully showing her mother ashamed of children and can not let go of the pain of Indignant Poem, and a mother in the era of war can not choose and have to To choose the pain. Return to the homeland is her dream, but she can not face is the separation of mother and child, from the other side of the day, life and death of two vast emotional facts. As the mother in any case can not be done, but repeatedly shaken, did not expect twelve years of hope and wait, actually at the expense of maternal love at the expense of a mother is how cruel

\section{Music returned home but left home}

Cai Wenji could not bear to leave with his son, but her thoughts of the home is all the time, "sense of parents, lamenting infinite" is the best proof. In this very contradictory, she decided to abandon the second son, but also to return to the hometown of the motherland. She looked at her tears in tears, until she disappeared in the sky. In the "same generation" in the eyes of Cai Wenji to return to his homeland, she is lucky, they also hope that one day to return to their homeland. But they can not help but think of "欷歔" "sobbing" "horse for the effort to hesitate, the car is not turning," they desperate pain, even the horses are moved, do not want to move forward. Qu Yuan "Li Sao" "servant sorrow in Ma Huai Xi, curl up and not" [3]one, also expressed the poet in the soon to leave the motherland, the feeling of heaven and earth scene. This is the "Indignant Poem and indignation" in the expression of the emotions have the same purpose.

And two sub-farewell to the sense of destruction, this is difficult to expressly. However, the poet is still full of hope and fantasy back home, and make her disappointed, "both to the family to do, and again and again outside the city for the mountains, Ting Yu Sheng Jing Yi. Bones do not know who, from the cross-covered" The [1] full of longing with Cai Wenji once again fell into the abyss of sorrow, the courtyard of a barren, relatives have done, no one can hope, once the dream of thousands of back into a nightmare around. "Climbing far overlooking" a desolate, "wolf" wailing in the wild, so that "lonely life" "soul suddenly flies." [1] Here with those who "peer" "Mu I alone" shape the formation of fierce conflict, unlimited hope and the despair of the death of the formation of suspended gap, enough to make her collapse numerous times. Such a brutal reality, "for the complex strong interest, although the students talk about", [1] he could not bear to see, can not help but ask whether there is a reason to live. In the helpless and "others" reluctantly persuaded, and finally decided to die.

Cai Wenji night wandering outside the drift for twelve years there is no sense of mind, so have been dreaming that one day can return to his hometown, and for this faith, she throws his son to return home. Cao Cao in the reunification of the North, read and the old Jiao Yong, to "Jinbi" redemption Cai Wen Ji to return to the Han, and later to marry and Dong Si, for the marriage of two people happy, in the historical data is not known. However, from the "Indignant Poem and indignation" "life in the new, dedicated to $\mathrm{Xu}$ Li. Flowing into a despicable, often fear of donation waste. Life when the time, pregnant years old" section, probably can see life is not happy. 
Soon, Dong Si "committed to death", Cai Wenji in order to give yourself a little hope, or desperate to rescue this unfriendly husband. She even if the ragged, unkempt also met with Cao Cao, asked Cao Cao let Dong Si. Although she rescued Dong Si, but also physically and mentally exhausted. Regardless of Cai Wenji from what the scholarly door, but also a mortal, the demand is nothing more than ordinary people's life. In the context of the time, her expectations are difficult to achieve, like Hemingway, "the sun rises as usual," the hero Barnes and his wife, sad and helpless filled with life.

\section{"Indignant Poem and indignation" of the "poem express" complex}

Poetry is not only an art form, but also used to express the poet's ambition and an important means of emotion. As early as two thousand years ago, Confucius told his disciples: "' Poetry "can be happy, you can view, you can group, you can complain." [6] means that poetry has to stimulate emotions, observe the community, The effect of blasphemy.

The ancients of the "poem express" view, first seen in the "Shangshu Yao Dian": "poem words, song Yongyan, sound by Yong, law and harmony, eight tone grams of harmony, no phase wins Lun, God and. "[2] With the development of literature," poem "made, not only for the successor inherited, but also for the development of poetry pointed out the direction of thinking. "Poems" of the rich connotation of the understanding of each person is not the same, different times the interpretation of their people are not the same. In general, "poem" itself not only includes ambition, politics and religion, history, etc., but also should contain "love". "The Book of Songs" not only includes the Western Zhou Dynasty sacrifice, the DPRK and other content, more "people" to express the emotional culture of space.

"Poetry" in the "Zhi" should be included in the original intention of both ambitions and emotions. "Love" focus on the inherent characteristics of poetry, "Chi" is focused on the performance of external, the two links together through a common link to promote the poetry forward. "Poem" is very strong "poem express" complex, the poet to narrative poetic expression, through the description of three time periods, to the reader to show a life in the troubled times in the hearts of the ill-fated beauty of the great Indignant Poem.

At the beginning of this poem, to explain the cause of Cai Wenji tragic encounter, that is, "Han lost the right handle, Dong Zhuo chaos". [1] Subsequently, a large number of Qiang soldiers east, "cut cut no relics" "slightly million", she is also looting in the ranks. On the way to the south of the Southern Huns, she was humiliated. The former bustling city, this "know the death", the people displaced, "corpses refused." Those who survived the people who have to be swept as slaves, life, such as grass mustard so that "every sentence death prisoners." This is what she suffered the spirit and the body by the double blow, and she thus express the helplessness and Indignant Poem, is the "poetry" in the article reflected.

The second part of the main writing Cai Wenji was forced to marry and the Southern Huns Zuoxian Wang, although the body to the wild, but all the time not miss the country. When she heard that the envoys were coming, let yourself be in the midst of despair in twelve years, and again with hope, "have to be free, and to rebuild your son." But, when the "children before holding my neck," the moment and heart hesitation, so that "collapse of five" "crazy crazy", and ultimately still difficult to go to Han's determination. "Love" is mainly embodied in the core characteristics of poetry, Cai Wenji to "return to the Han" and the tactics of the conflict and burst out of the special feelings, deeply hurt the poet's deepest heart, and the poetic "Love" to a climax.

The third part is mainly to write Cai Wen Ji full of hope and joy, back home country. But the sight of the front but her "soul suddenly flew." "Family to do" "Ting Yu Sheng Jing Ai" "bones do not know who" scene in the mind kept flashing, all this makes her "lonely life". She did not choose to death as a relief, and only a trace of hope "to life in the new". However, the "Indignant Poem" of the last sentence of "life when the time, worry age", [1] or out of which the outcome and destination. This is both a poet's question, but also helpless sigh. 


\section{References}

[1] Fan Ye. Later Han [M]. Beijing: Zhonghua Book Company, 1965: 2800.

[2] Chen Qizhi. Chinese ancient literary essence of translation [M]. Changsha: Hunan Publishing House, 1993.

[3] Fu Gang, Yan Qi. Selected Works of Chinese Literature [M]. Beijing: Zhonghua Book Company, 2007.

[4] Wang Guowei, Teng Xianhui. New words of human words [M]. Jinan: Qilu Publishing House, 2001.

[5] Cheng Junying. Poetry translation [M]. Shanghai: Shanghai Ancient Books Publishing House, 2014.

[6] Lai Kehong. The Analects of Confucius [M]. Shanghai: Fudan University Press, 2000. 